\title{
BMJ Open Outcomes of disease prevention and management interventions in food pantries and food banks: a scoping review
}

Christopher R Long, ${ }^{\oplus 1}$ Brett Rowland, ${ }^{2}$ Susan C Steelman, ${ }^{3}$ Pearl A McElfish ${ }^{1}$

To cite: Long CR, Rowland B, Steelman SC, et al. Outcomes of disease prevention and management interventions in food pantries and food banks: a scoping review. BMJ Open 2019;9:e029236. doi:10.1136/ bmjopen-2019-029236

- Prepublication history for this paper is available online. To view these files, please visit the journal online (http://dx.doi. org/10.1136/bmjopen-2019029236).

Received 18 January 2019 Revised 08 July 2019 Accepted 31 July 2019
Check for updates

(C) Author(s) (or their employer(s)) 2019. Re-use permitted under CC BY-NC. No commercial re-use. See rights and permissions. Published by BMJ.

${ }^{1}$ College of Medicine, University of Arkansas for Medical

Sciences Northwest, Fayetteville, Arkansas, USA

${ }^{2}$ Office of Community Health and Research, University of Arkansas for Medical Sciences Northwest, Fayetteville, Arkansas, USA

${ }^{3}$ Division of Academic Affairs, University of Arkansas for Medical Sciences, Little Rock, Arkansas, USA

Correspondence to Dr Christopher R Long; crlong2@uams.edu

\section{ABSTRACT}

Objective Food insecurity affects millions of Americans and is associated with a range of adverse health outcomes. Food insecure individuals often obtain food from food pantries/banks, prompting health researchers to implement disease prevention/management interventions at these sites. This review examined the existing peer-reviewed research on disease prevention/ management interventions implemented in food pantries/ banks.

Design Scoping review.

Data sources Databases searched included MEDLINE, Web of Science, CINAHL and Cochrane. Search strategies included Medical Subject Headings and key terms, including food pantry, food bank, food shelf, food aid and related concepts.

Eligibility criteria Studies were included if they described an intervention involving food pantries/banks where at least one biometric indicator was included as an outcome variable. Articles focused solely on the quality of foods distributed, the diet quality of food pantry/bank clients or government food aid programmes were excluded.

Data extraction and synthesis Extracted data included publication details, intervention type, study design, participant characteristics, study outcomes, and barriers and facilitators of intervention implementation.

Results A total of 3317 articles were assessed for eligibility. Six studies met the predefined inclusion criteria. The studies employed a range of intervention approaches to manage or prevent a number of chronic diseases, including obesity, type 2 diabetes and HIV. The studies examined a range of biometric outcomes, including body mass index, glycated haemoglobin and blood pressure. Information about the food pantries/banks where the interventions were conducted was lacking. The studies documented multiple barriers and facilitators related to costs, sustainability and organisational capacity. Conclusions To our knowledge, this is the first review to examine disease prevention and management interventions conducted in food pantries and food banks. Given the high number of households who obtain food from food pantries/banks and the chronic health conditions associated with food insecurity, this review highlights the need for more high-quality research in this setting.
Strengths and limitations of this study

- This scoping review was guided by a previously published peer-reviewed protocol.

- To our knowledge, this is the first review to examine disease prevention/management interventions in food pantries/banks.

- This review is based on rigorous searches of peer-reviewed literature, but it did not include grey literature searches.

- Exclusion of food pantry/bank studies that lacked a biometric outcome may have limited the findings regarding barriers and facilitators to intervention implementation.

\section{INTRODUCTION}

Food insecurity affects the well-being of many people in the USA. Defined as lack of access to sufficient amounts of nutritious food, food insecurity affected 15 million US households (11.8\% of US households) in $2017 .{ }^{1}$ When faced with food insecurity, many of these households obtain food from food pantries and food banks.

Food pantries are local emergency food organisations that provide aid to food insecure households through the distribution of unprepared food for offsite consumption. ${ }^{2}$ Food pantries are often associated with faithbased organisations and typically rely on funding from local donors and labour from unpaid volunteers. ${ }^{2} 3$ Food banks are organisations that distribute food to food pantries and other organisations that distribute food directly to households or individuals. ${ }^{3}$ (There is much regional variation in the use and meanings of the terms food pantry and food bank. For this reason, the present review uses the definitions typical of US Department of Agriculture (USDA) reporting. ${ }^{2}$ ) In 2017, $4.7 \%$ of US households-and $26.0 \%$ of food insecure households-acquired food from 
food pantries, ${ }^{2}$ many of which obtained food from food banks. ${ }^{4}$

In addition to lack of access to sufficient food, food insecure households face many health challenges. ${ }^{5}$ Food insecurity is associated with increased healthcare utilisation and costs for working-age adults. ${ }^{6}{ }^{7}$ Insufficiently nutritious dietary patterns are associated with a range of chronic health conditions, including obesity, cancer and cardiovascular disease. ${ }^{89}$ In particular, food insecurity is related to increased risk for many chronic health conditions, including diabetes, ${ }^{10-13}$ obesity, ${ }^{14-16}$ dyslipidaemia $^{17} 18$ and hypertension. ${ }^{12} 1317$ Moreover, food insecurity is related to less effective management of many health conditions, including depression, ${ }^{19} 20$ diabetes, ${ }^{112021}$ hypertension ${ }^{20} 22$ and HIV. ${ }^{20}{ }^{23}$ Likewise, when an adult in the household has at least one chronic physical or mental health condition, risk for household food insecurity increases. ${ }^{24} 25$

Because of the health difficulties associated with food insecurity and because many food insecure households obtain food from food pantries and food banks, health researchers have begun to work with food pantries and food banks as sites to implement and evaluate disease prevention and management interventions. ${ }^{26-28}$ Food pantries and food banks may represent an opportunity to intervene with food insecure households who otherwise may not be easy to reach at more traditional intervention sites (eg, worksites, hospitals, schools or churches).$^{29}$ In contrast to more traditional intervention sites, there have been relatively few published studies of disease prevention and management studies implemented in food pantries and food banks. For this reason, a scoping review was conducted to assess what has been learned about this topic and to identify remaining gaps in knowledge.

The purpose of this scoping review was to examine existing research on disease prevention and management interventions implemented in food pantries and food banks. Other reviews have examined studies documenting food pantry and food bank clients' diet quality and the nutritional quality of food pantries' inventories of food. ${ }^{30}{ }^{31}$ However, to our knowledge, there have been no reviews focused directly on studies that specifically incorporate biometric indicators closely associated with the diagnosis or progression of disease, including biometric measures of body weight, body mass index (BMI), glycated haemoglobin (HbA1c) and blood pressure. These biometric indicators provide relatively objective measures of health impact beyond self-reported measures and are typically reported in disease prevention and management intervention trials. ${ }^{32}{ }^{33}$ For these reasons, this scoping review focused on studies that reported biometric outcomes of disease prevention and management interventions implemented in food pantries and food banks. In particular, this review focused on the following questions:

1 . What were the primary biometric indicators targeted by the disease prevention and management interventions implemented in food pantries and food banks, and for which biometric indicators did these interventions show success?

2. Did the success of these interventions vary as a function of sex, ethnicity or other demographic characteristics of participants?

3. What barriers and facilitators to successful implementation of these interventions were reported?

\section{METHODS}

This scoping review followed applicable principles described in the Preferred Reporting Items for Systematic Reviews and Meta-Analyses (PRISMA), PRISMA-Protocols (PRISMA-P) and PRISMA-Extension for Scoping Reviews (PRISMA-ScR) statements. ${ }^{34-37}$ The protocol for this scoping review was developed using the methodological framework developed by Arksey and $\mathrm{O}^{\prime} \mathrm{Malley}^{38}$ and advanced by Levac et $a l^{39}$ and is published elsewhere. ${ }^{40}$

\section{Eligibility criteria}

Eligibility criteria were defined according to the PICOTS (population, intervention, comparator, outcomes, timing and setting) framework. ${ }^{41-43}$ These criteria were specified in the published protocol as follows:

- Participant population: members of any racial/ethnic, sex, or age group who use a food pantry or food bank.

- Intervention type: all types of interventions focused on disease prevention or management among food pantry or food bank clients (eg, with respect to diabetes, obesity, infectious diseases).

- Comparator: no intervention, other intervention or within-participant preintervention.

- Outcomes: studies measuring and reporting at least one biometric indicator (eg, BMI, blood pressure, blood glucose) as an intervention outcome variable.

- Context: interventions taking place in a food pantry or food bank or having a central component taking place in a food pantry or food bank.

- Study type: all types of studies (eg, randomised controlled trials, pilot studies, case studies).

No exclusions were made regarding duration of intervention, follow-up schedule, country or region. Studies were required to be peer-reviewed, published in English and published in 1997 or later. Studies describing interventions focused only on improving the quality of foods distributed by food pantries/banks or studies describing the diet quality of food pantry/bank clients were excluded. Likewise, studies focused on government assistance programmes (eg, USDA's Supplemental Nutrition Assistance Program; Special Supplemental Nutrition Program for Women, Infants, and Children; or National School Lunch Program) were excluded.

\section{Information sources}

Biomedical databases were searched on three different vendor platforms. To ensure availability of advanced search features, MEDLINE and MEDLINE In Process \& Daily Updates were searched via the OVID platform. Detailed evidence-based medicine databases were also 
searched via OVID and included the All EBM Reviews collection of databases which cover ACP Journal Club, Cochrane Central Register of Controlled Trials, Cochrane Database of Systematic Reviews, Cochrane Methodology Register, Databases of Abstracts of Reviews of Effects, Health Technology Assessment and the NHS Economic Evaluation Database. Also searched were the Cumulative Index to Nursing and Allied Health Literature-CINAHL Complete (EBSCO) and Science Citation Index and Social Sciences Citations Index, both via Web of Science.

\section{Search strategy}

As described in the published protocol, the authors identified all pertinent concepts and consulted on specific terminology. All search strategies were created by medical librarian coauthor (SCS) in consultation with coauthors (CRL and BR) and run in October and November 2017. Controlled vocabulary terms were combined with advanced textword search techniques including adjacency, nesting and truncation. Specific Medical Subject Headings (MeSH) use and exploded (to incorporate more specific headings under the MeSH terms) included Food Assistance/, Food/, Food Supply/ and Hunger/. The main concepts and phrases searched included food aid, food bags, food banks, food pantries, food shelves, soup kitchens, hunger and combinations for food insecurity. Disaster-related food services surrounding tornados, hurricanes, fires, mudslides, floods, poisonings or spoilage were not included; however, the overall concept of emergency food aid was searched. The main MEDLINE strategy was adapted to include appropriate controlled vocabulary headings and textword searching combinations as required for specific databases and vendor platforms. All final strategies covered 1997-2017 and were limited to English language. From November 2017 to August 2018, auto alerts were implemented for the MEDLINE database and new items resulting from the original MEDLINE search strategy were reviewed by the team for possible inclusion. The exact MEDLINE strategies for the OVID platform are shown in table 1 and were revised only slightly from those published in the protocol (eg, the exclusionary term 'hurricane*' was added in Search \#6, and the term 'scarcity' was added in Search \#9). Final strategies from all other databases may be requested from the corresponding author (CRL).

\section{Study selection process}

The searched databases had overlap in their indexed journal titles, which led to duplicate records. All records (which included article titles and abstracts) from each database were exported to RefWorks (V.2.0), a web-based bibliographic management tool. ${ }^{44}$ A coauthor (SCS) used RefWorks' duplicate-check feature and conducted a manual review of each record removed. The RefWorks folder of results was then exported into Rayyan, a free web application designed to expedite screening of titles and abstracts, and shared with all coauthors. ${ }^{45}$ As additional duplicates were identified via Rayyan's duplicate feature, they were manually deleted from the Rayyan database by a coauthor (BR.). Throughout the study selection process, coauthors also performed manual searches (via Google

\section{Table 1 Detailed Ovid MEDLINE search strategy}

\begin{tabular}{|c|c|c|}
\hline $\mathbf{N}$ & Searches & Results \\
\hline 1 & $\begin{array}{l}\text { exp Food Assistance/ and (aid or aide or assist* or bag* or bank* or box }{ }^{\star} \text { or pantr }{ }^{\star} \text { or shelf or shelves } \\
\text { or secure or insecurity or insecure).ti,ab. }\end{array}$ & 301 \\
\hline 3 & $\begin{array}{l}\text { exp Food Supply/ and (food adj2 (aid or aide or assistance* or bag* or bank* or box* or pantr* or shelf } \\
\text { or shelves)).ti. }\end{array}$ & 126 \\
\hline 5 & $\begin{array}{l}\text { (food adj2 (aid or aide or assist* or bag* or bank* or box* or pantr* or shelf or shelves or secure or } \\
\text { insecurity or insecure or scarcity)).ti. }\end{array}$ & 1121 \\
\hline 6 & $\begin{array}{l}\left(\left(\text { emergency adj2 }\left(\text { food }^{\star} \text { or meal }{ }^{\star} \text { or nutrition)) not (disaster }{ }^{\star} \text { or tornado* or hurricane* or fire }{ }^{\star} \text { or }\right.\right.\right. \\
\left.\left.\text { mudslide* or flood }^{\star} \text { or poisoning or spoil }{ }^{\star}\right)\right) \cdot \mathrm{mp} \text {. }\end{array}$ & 132 \\
\hline 9 & $\begin{array}{l}\text { exp Hunger/ and (food adj2 (aid or aide or assist* or bag* or bank* or box* or pantr* or shelf or shelves } \\
\text { or secure or insecurity or insecure or scarcity)).ti. }\end{array}$ & 178 \\
\hline 10 & 1 or 2 or 3 or 4 or 5 or 6 or 7 or 8 or 9 & 2053 \\
\hline 11 & limit 10 to English language & 1976 \\
\hline 12 & limit 11 to $y r=" 1997-2017 "$ & 1794 \\
\hline
\end{tabular}




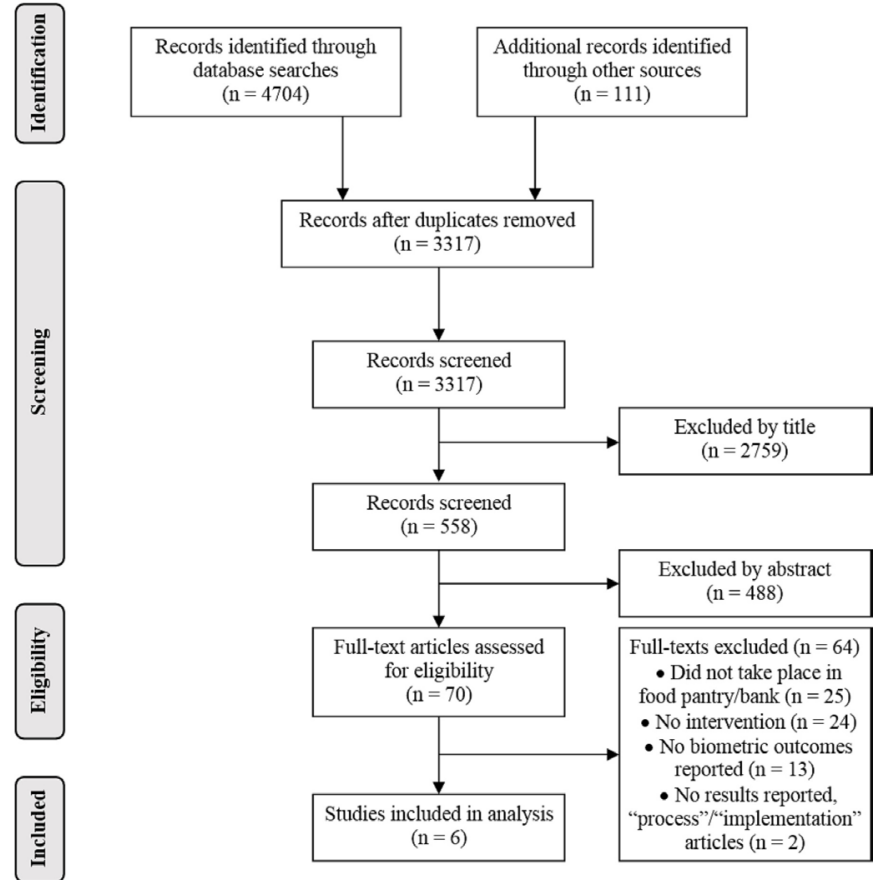

Figure 1 Modified Preferred Reporting Items for Systematic Reviewsand Meta-Analyses flow diagram.

Scholar) and manual examinations of relevant articles' reference lists.

Each de-duplicated record (ie, study title and abstract) was reviewed by either of two reviewers (BR and CRL) to determine whether the study met eligibility criteria. For each study judged eligible or judged uncertain by either reviewer, each reviewer reviewed the article's full text to verify eligibility. In the few instances in which discrepancies arose between the reviewers' determination of a study's eligibility, these discrepancies were resolved through discussion between the reviewers.

\section{Data extraction process and data items}

Following the process described in the study protocol, the two reviewers used a Microsoft Excel (V.15.0) spreadsheet developed for this study to extract relevant data from the included articles. Each reviewer extracted data from each eligible study; extracted data were compared, and no major discrepancies were identified.

The reviewers extracted the following data items from each eligible article:

- Participant population: race/ethnicity, sex, age group, urban/rural, any other participant characteristics used as an inclusion criterion.

- Intervention type: stated disease focus of intervention (eg, prevention or management of diabetes, obesity or infectious diseases), duration of intervention and brief description of intervention.

- Comparator: comparator type (eg, no intervention or within-participant preintervention) and description.

- Outcomes: biometric outcomes (eg, BMI, blood pressure or blood glucose), any other outcomes reported and the extent to which each outcome was affected by the intervention.

- Context: whether the intervention took place in a food pantry or food bank and other notable characteristics of the food pantry or food bank.

- Barriers and facilitators to successful intervention implementation: barriers or facilitators of intervention implementation noted by study authors, including factors related to intervention characteristics, implementation setting, individuals involved and implementation process. ${ }^{46}$

- Study type: type of study design used (eg, randomised controlled trial, single-arm pilot study or single-subject design).

- Publication details: authors, article title, journal title, year of publication, volume number, issue number, page numbers.

\section{Data synthesis}

For most extracted data fields, data synthesis involved producing quantitative descriptive summaries (eg, frequencies). Coverage and gaps in existing literature were further assessed using qualitative summaries based on inductive coding of the extracted data. These focused on identifying diseases targeted by the studies' interventions, the studies' primary outcomes and results, and barriers and facilitators to implementation of the interventions.

\section{Patient and public involvement}

There was no involvement of patients or the public in this scoping review.

\section{RESULTS}

The systematic database searches produced 4704 total records. Automatic MEDLINE updates, manual searches (via Google Scholar) and manual examinations of article reference lists produced an additional 111 records. After duplicates were removed, 3317 records were reviewed for inclusion. Based on title or abstract, 3247 were excluded. Seventy full-text articles were retrieved and reviewed for inclusion. Ultimately, six studies fulfilled the predetermined eligibility criteria. ${ }^{47-52}$ Figure 1 summarises the detailed screening process.

\section{Characteristics of included studies}

Basic characteristics of the six studies are shown in table 2. Four of the six studies were quasi-experimental, single-arm pre/post studies. ${ }^{47}$ 49-51 Two studies randomised participants to intervention or control groups and analysed differences in outcomes between the two arms. ${ }^{4852}$ Four of the six interventions took place over the course of 6 months, ${ }^{480-52}$ one intervention took place over 3 months ${ }^{47}$ and one lasted 6 weeks. ${ }^{49}$ Time ranges from enrollment/baseline to final data collection follow-ups were 3 months ${ }^{47}$ and 6 months. ${ }^{4-52}$ The studies focused explicitly on the prevention and/or management 


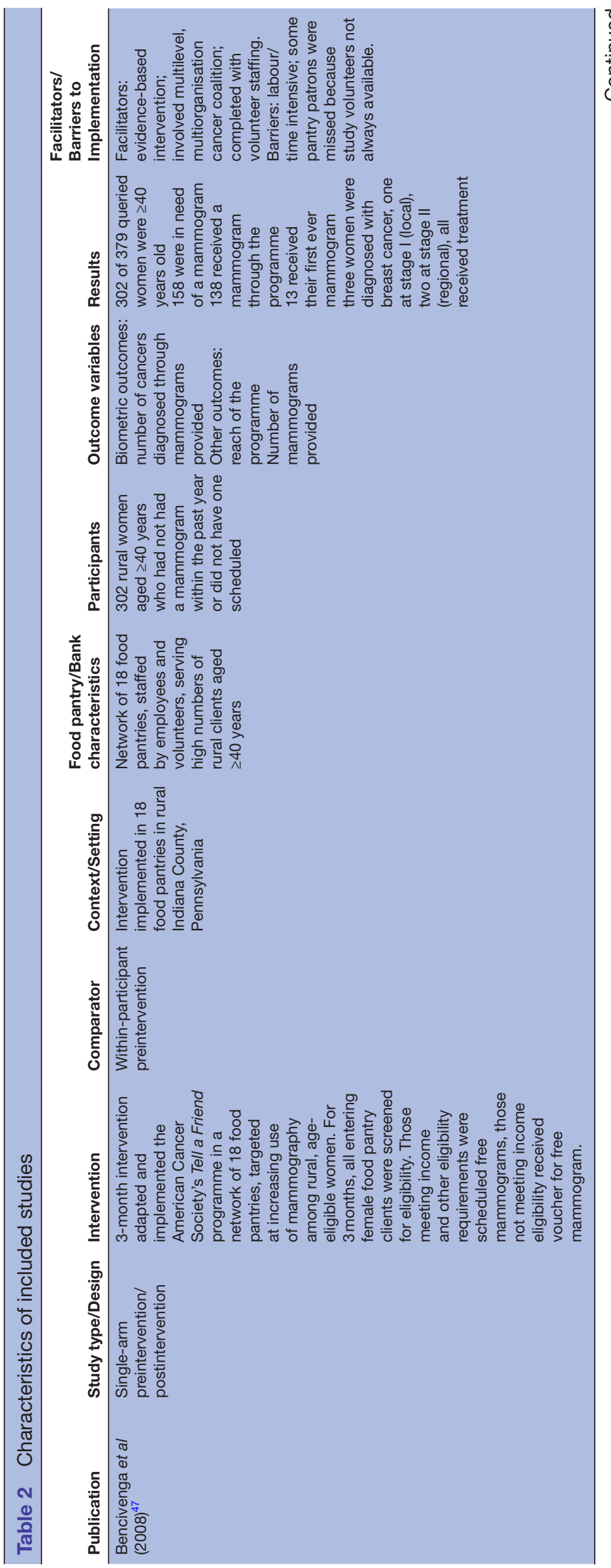

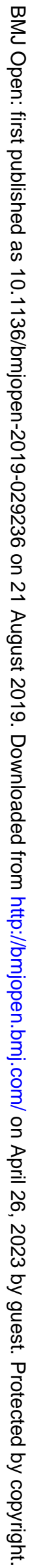




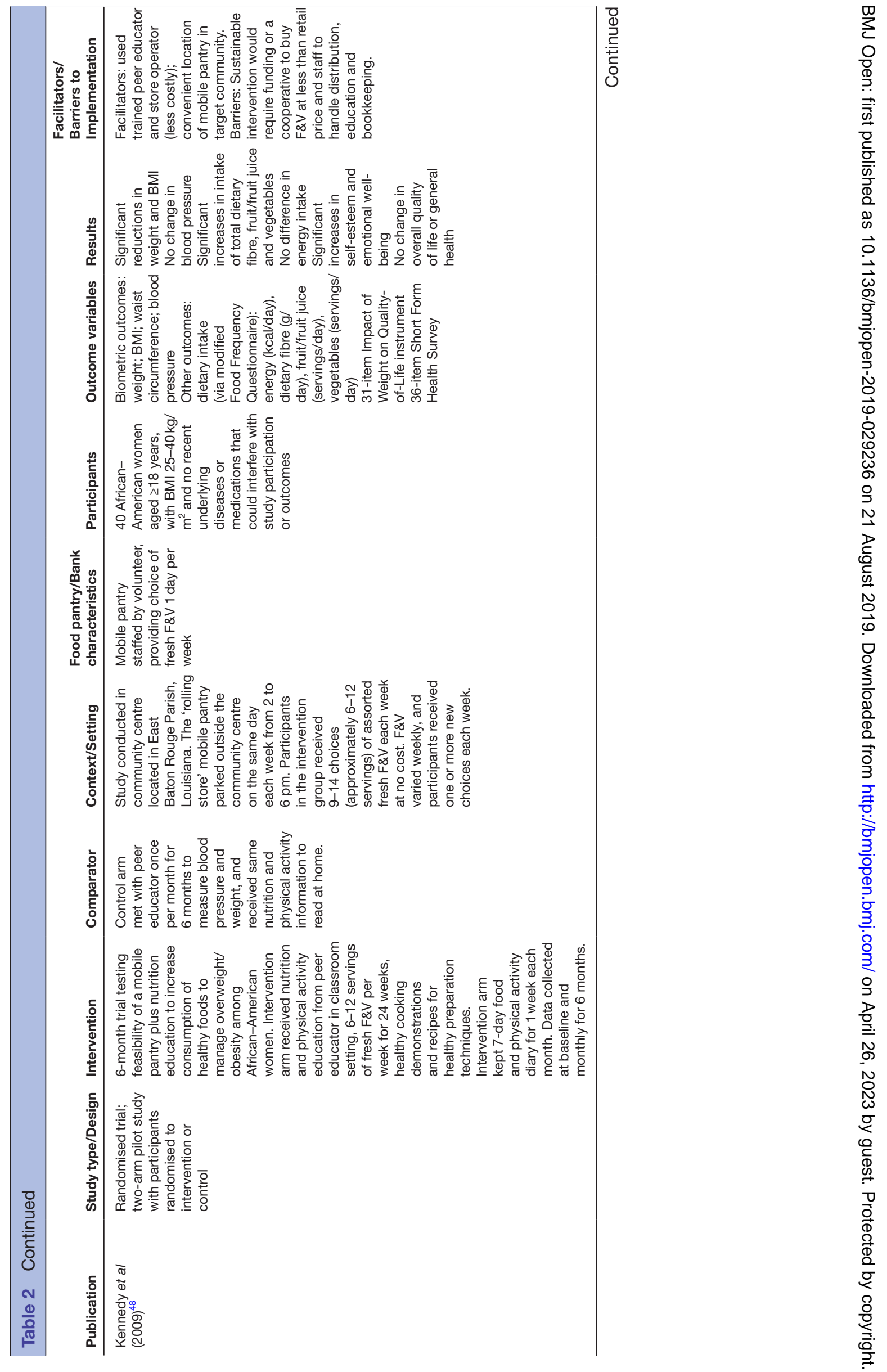




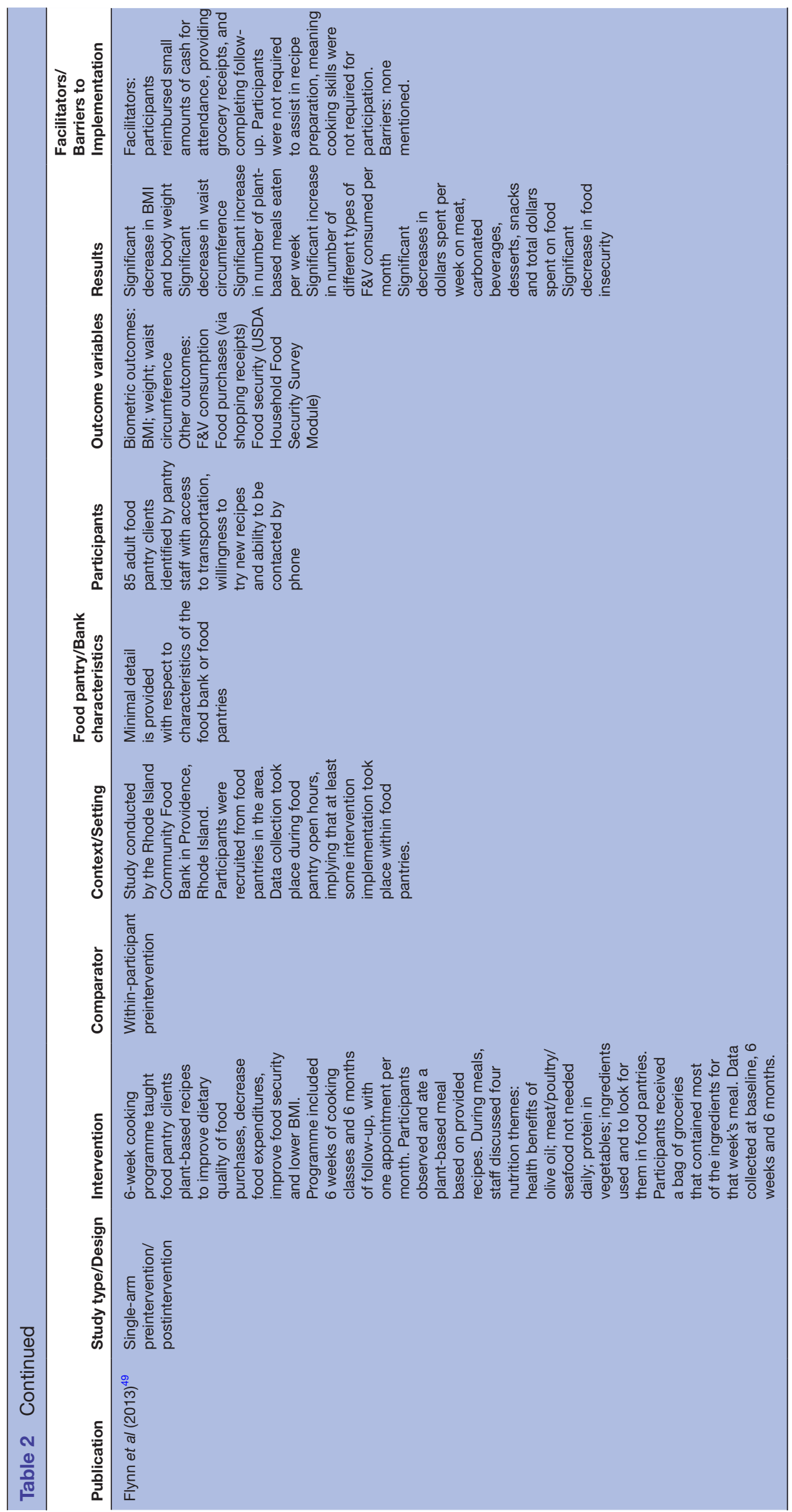




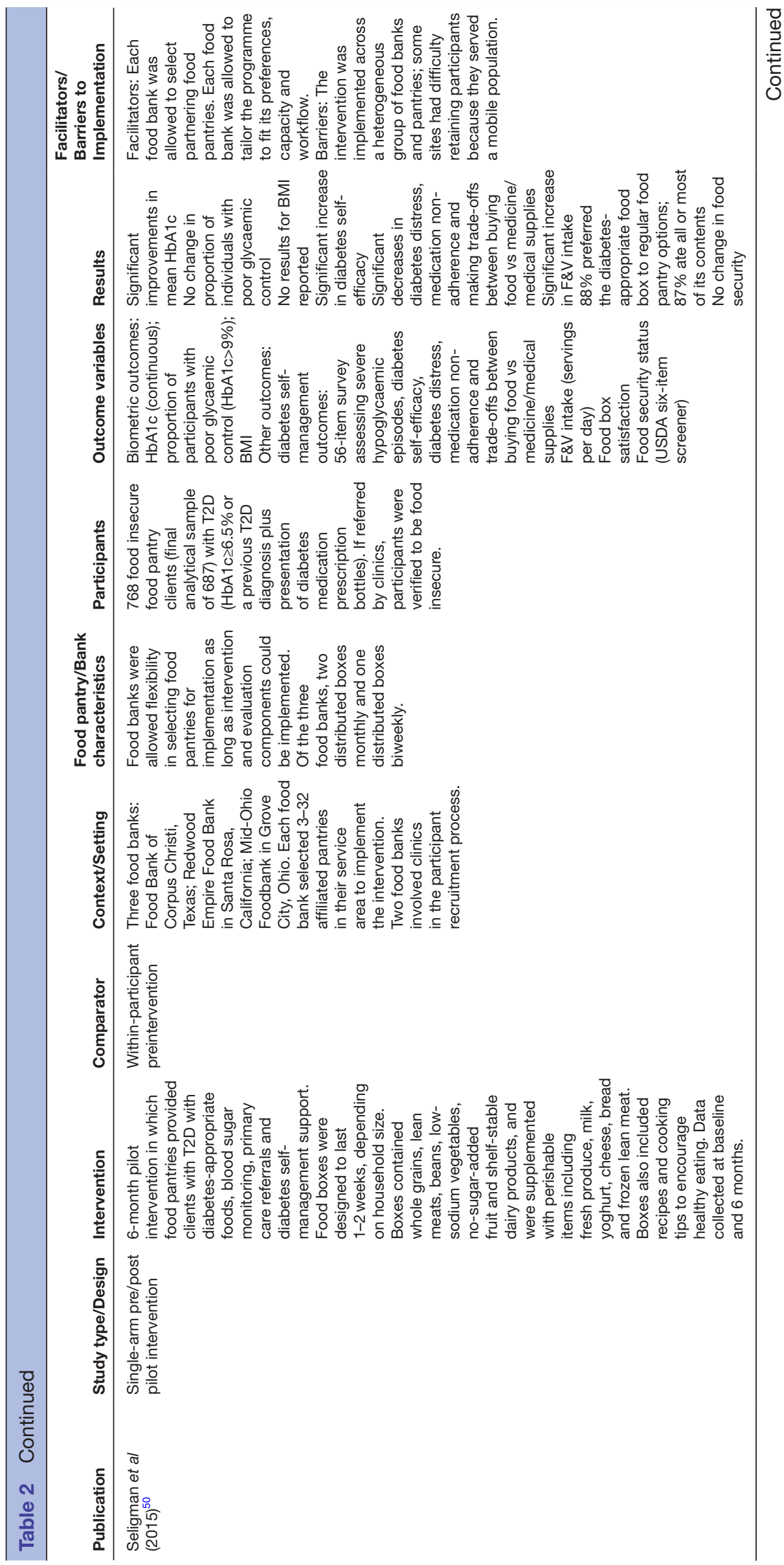

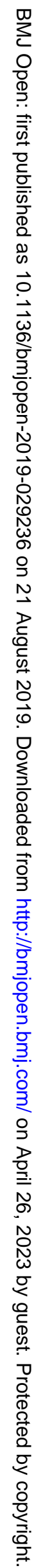




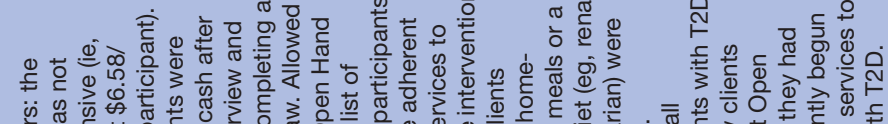

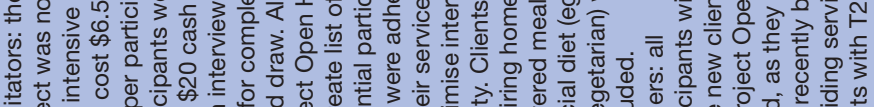

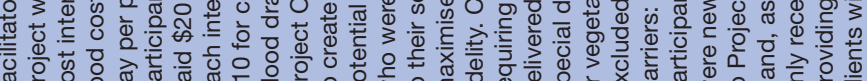

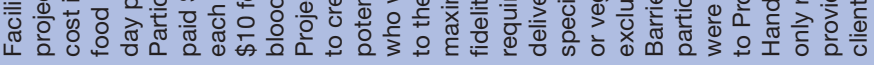

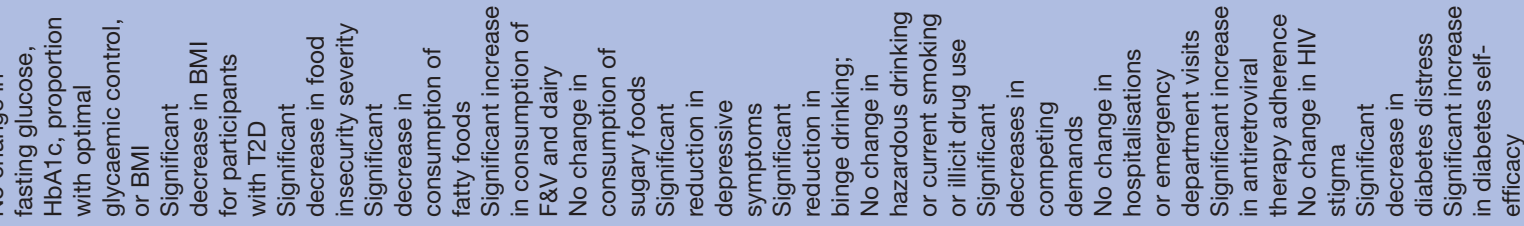

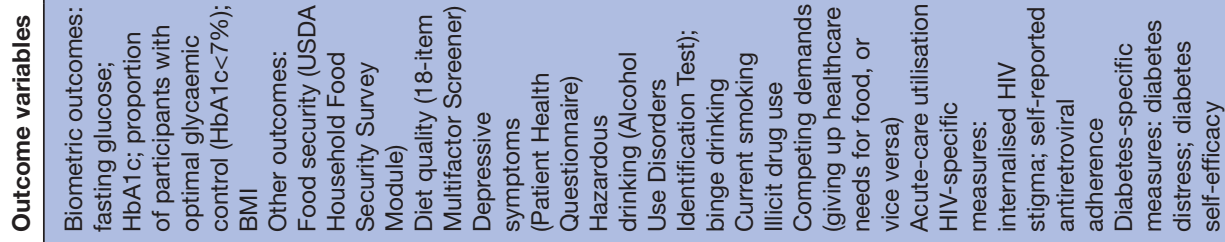

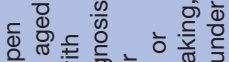

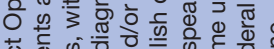

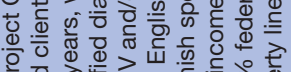

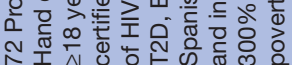

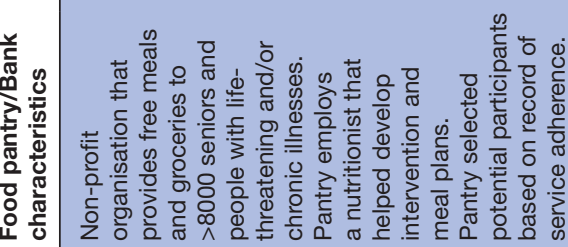

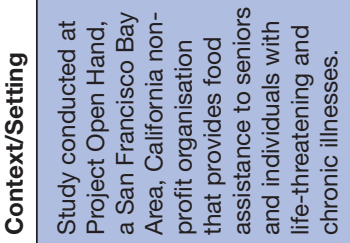

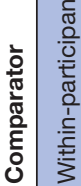

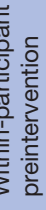

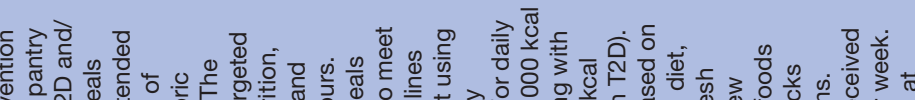

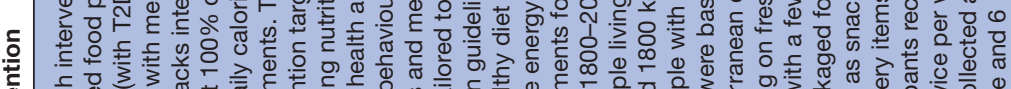

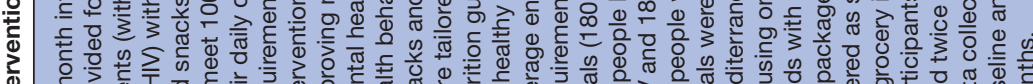

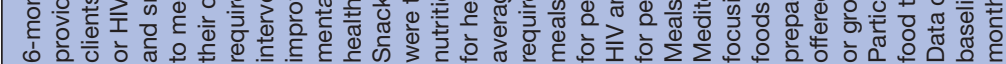

क्ञ

落

$\stackrel{2}{9} \div$

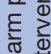

离.

ס

के

ن

$\frac{\sqrt{2}}{\frac{0}{0}}$

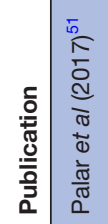




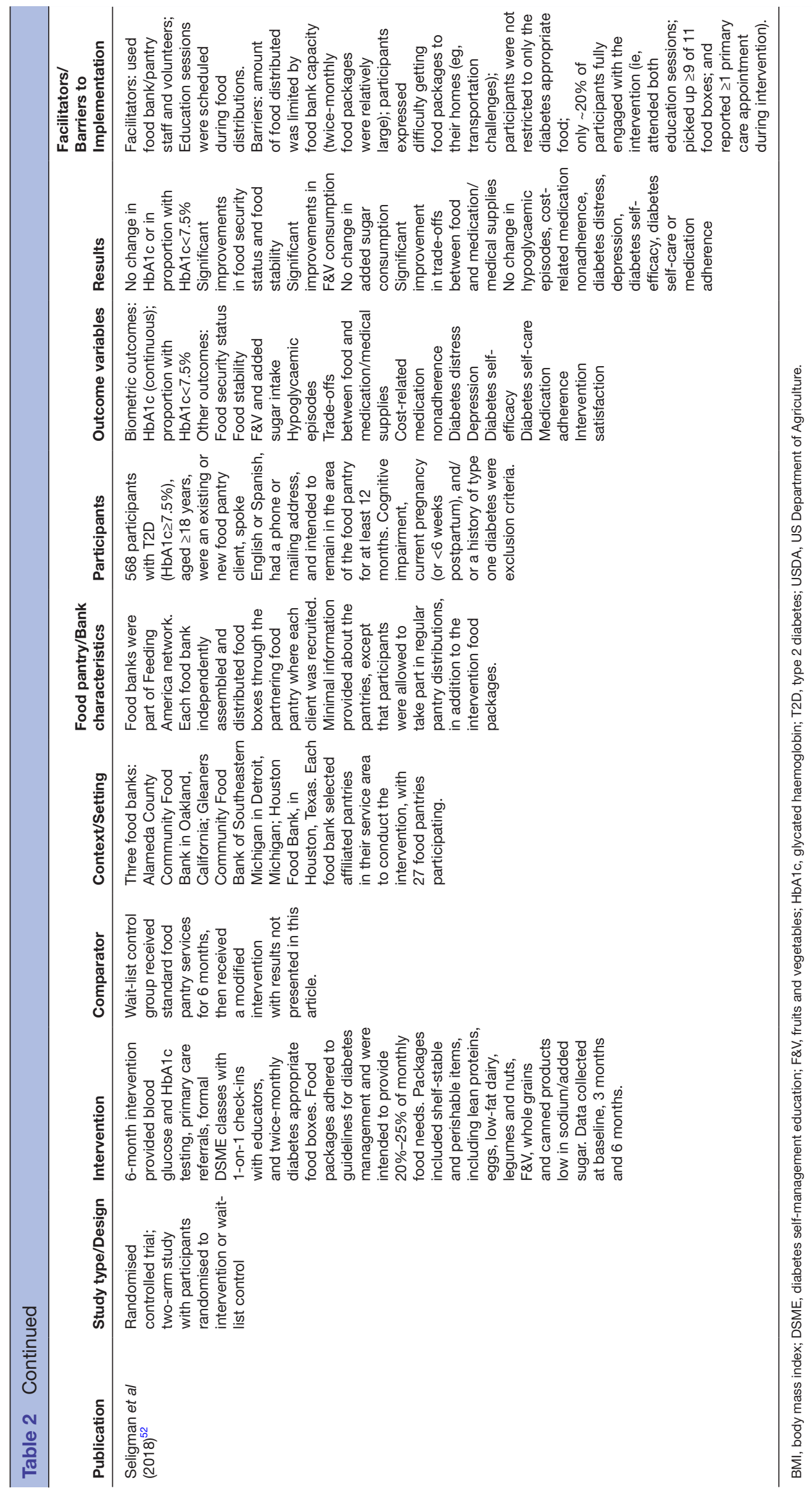


of specific chronic diseases, including type 2 diabetes, ${ }^{50-52}$ overweight/obesity, ${ }^{48}{ }^{49}$ cancer $^{47}$ and HIV. ${ }^{51}$ Four studies focused on providing education (eg, nutrition education, physical activity education, diabetes self-management education) in combination with providing foods that support a healthy diet (eg, fresh fruits and vegetables, lean meats, whole grains) ${ }^{48-50} 52$ Another study focused solely on providing foods that support a healthy diet, ${ }^{51}$ and the remaining study focused solely on increasing participation in mammography. ${ }^{47}$ Sample sizes of the six studies at baseline/preintervention ranged from 40 to 768 , with a mean of 305.8 and a median of 193.5. All studies took place in the USA.

\section{Results of included studies}

\section{Study populations}

Consistent with this scoping review's eligibility criteria, the studies' participant populations comprised food pantry and/or food bank clients. The majority of participants in the studies were women; two studies included only women, ${ }^{47} 48$ while only one study included more men than women. ${ }^{51}$ Across the studies, participants' mean ages ranged from 45.9 to 56.6 years. The studies varied considerably with regard to participant race/ethnicity. One study included only African-Americans ${ }^{48}$; in one study, the majority of participants were white ${ }^{49}$; two studies' samples were predominantly Hispanic/Latino ${ }^{50} 52$; one study was split majority white/black ${ }^{51}$; and one study did not report participants' races/ethnicities. ${ }^{47}$ Four of the six studies required that participants had a specific chronic disease diagnosis for inclusion. Of these, two studies required that participants had a type 2 diabetes diagnosis ${ }^{50} 52$; one required an HIV and/or type 2 diabetes diagnosis ${ }^{51}$; and another used presence of overweight/obesity as an inclusion criterion (BMI 25-40). ${ }^{48}$ Flynn et $a l$ s study inclusion criteria did not implicate a specific chronic disease, limiting inclusion to food pantry clients who had access to transportation and a working phone and who were willing to try new recipes. ${ }^{49}$ However, participants had a mean BMI of 33.3 at baseline and the intervention focused on increasing consumption of vegetables/plant-based meals and decreasing purchases of less healthy foods, indicating a focus on overweight/obesity. ${ }^{49}$

\section{Use of food pantries and food banks in interventions}

Consistent with this scoping review's eligibility criteria, at least one component of each included study's intervention took place in a food pantry or food bank. For most of these studies, the published articles were focused on describing the interventions rather than the food pantries/banks themselves. For this reason, information about the food pantries/banks where the interventions were conducted was often lacking. None of the studies fully described the food pantry or food bank operations for all sites, including geographical location, their organisational structure (eg, situated in a church or other faith-based organisation vs a standalone non-profit) and food distribution model (eg, client choice vs pre-boxed/ bagged).

With respect to the food pantry/bank components of the interventions, one study simply added a healthier food distribution to regular food pantry services that were available to clients. ${ }^{52}$ Additionally, three studies described providing education to food pantry clients on-site at the food pantries. ${ }^{495052}$ One study was conducted in a local community centre and employed a mobile food pantry to provide food to clients as part of the intervention. ${ }^{48}$ (Although they referred to this mobile pantry as a 'rolling store' throughout the article, the 'store' meets the definition of a food pantry specified in this review's protocol, as the mobile pantry provided participants in the study with free fresh fruits and vegetables to be consumed at home every week for 24 weeks.)

Three studies involved both food pantries and food banks to recruit participants and conduct their interventions. ${ }^{49} 5052$ These three studies relied on food banks to identify food pantries in the food banks' service networks that would be able to support the needs of the respective interventions.

\section{Biometric outcomes}

Four studies examined changes in BMI. ${ }^{48-51}$ Three studies examined changes in HbAlc as a continuous outcome, as well as the proportion of participants that achieved/ failed to achieve glycaemic control (although the specific criteria for achieving glycaemic control varied among studies). ${ }^{50-52}$ Two studies examined changes in body weight (separate from BMI) and waist circumference. ${ }^{48} 49$ Measures of blood pressure, ${ }^{48}$ fasting glucose ${ }^{51}$ and diagnosed cancers ${ }^{47}$ were reported in one study each.

Studies analysing changes in body weight and/or BMI showed mixed results. Kennedy et al found significant reductions in weight and BMI from baseline to 6 months in the mobile pantry intervention group relative to the control group. ${ }^{48}$ The intervention group's mean weight decreased by $2.0 \mathrm{~kg}$, while the control group's mean weight increased by $1.1 \mathrm{~kg}(\mathrm{p}<0.001)$. Likewise, the intervention group's mean BMI decreased by $0.7 \mathrm{~kg} / \mathrm{m}^{2}$, while the control group's mean BMI increased by $0.4 \mathrm{~kg} / \mathrm{m}^{2}$ ( $\mathrm{p}=0.001)$. Participants in Flynn et al's single-arm 6-week healthy cooking intervention showed significant reductions in BMI and weight at 6-month follow-up. ${ }^{49}$ Mean BMI decreased from $33.3 \mathrm{~kg} / \mathrm{m}^{2}$ at baseline to $32.9 \mathrm{~kg}$ / $\mathrm{m}^{2}$ at 6 months $(\mathrm{p}=0.05)$, and the mean change in weight was a decrease of $1.44 \mathrm{~kg}$. The medically appropriate food support study conducted by Palar et al produced no significant changes in BMI between baseline and 6-month follow-up across all participants; however, BMI was significantly reduced among the subset of participants with type 2 diabetes $\left(36.1 \mathrm{~kg} / \mathrm{m}^{2}\right.$ at baseline vs $34.8 \mathrm{~kg} / \mathrm{m}^{2}$ at follow-up; $\mathrm{p}=0.035) .{ }^{51}$ Seligman et al reported collecting BMI at baseline, but either did not collect follow-up BMI or did not report findings. ${ }^{50}$

Among the three studies reporting HbAlc and/or fasting glucose as a measure of glycaemic control, only 
one study reported a statistically significant change. ${ }^{50}$ For all participants in the diabetes-appropriate food and self-management support intervention who gave baseline and 6-month follow-up HbA1c samples $(\mathrm{n}=768)$, Seligman et alfound a mean $0.15 \%$ (NGSP unit) reduction in HbAlc $(\mathrm{p}<0.01) .^{50}$ Similarly, among participants with poorly controlled HbA1c $(\geq 7.5 \%)$ at baseline $(\mathrm{n}=411)$, the study reported mean reductions in $\mathrm{HbAlc}$ of $0.48 \%$ (NGSP unit) between baseline and 6-month follow-up $(p<0.001)$. None of the three studies that assessed changes in the proportion of participants with poor glycaemic control/ optimal glycaemic control reported significant changes in this measure. ${ }^{50-52}$

Results were mixed for studies analysing changes in waist circumference. Flynn et als healthy cooking intervention reported a significant decrease in waist circumference for the $86 \%$ of participants $(\mathrm{n}=54)$ with both baseline and 6-month follow-up measurements $(96.2 \mathrm{~cm}$ vs $95.3 \mathrm{~cm}$; $\mathrm{p}=0.05) .{ }^{49}$ Alternatively, there were no significant changes observed in waist circumference or blood pressure from baseline to 6 months in Kennedy et al $l$ s mobile food pantry intervention. ${ }^{48}$ Bencivenga et al $l$ s mammogram promotion study showed that 3 of 138 women who received a mammogram as a result of the food pantry-based intervention were diagnosed with cancer. ${ }^{47}$

\section{Other outcomes}

In addition to biometric outcomes, five studies described changes in participants' self-reported intake of specific foods, including significant increases in intake of vegetables, fruit/fruit juice, dietary fibre, dairy and plantbased meals in general, as well as significant decreases in consumption of fatty foods. ${ }^{48-52}$ Additionally, Flynn $e t$ $a$ l found a significant decrease in the amount of money participants spent on unhealthy foods between baseline and 6-month follow-up, including decreases in participants' spending on meats, carbonated beverages and desserts. ${ }^{49}$

Three studies found decreased food insecurity for participants from baseline to follow-up, ${ }^{49} 5152$ whereas one study found no change in participants' food insecurity. ${ }^{50}$ Specifically, Flynn et als s healthy cooking intervention reduced mean food insecurity scores from 3.2 at baseline to 2.07 at follow-up $(\mathrm{p}<0.01) .{ }^{49}$ Palar $e t$ al's medically appropriate food support intervention significantly reduced the proportion of individuals experiencing very low food security from baseline to 6-month follow-up $(59.6 \%$ vs $11.5 \%, \mathrm{p}<0.0001) .{ }^{51}$ Finally, in Seligman et al $\mathrm{s}$ trial, participants randomised to the diabetes self-management support and diabetes-appropriate food condition showed significant reductions in the proportion with low and very low food security, compared with the control group at 6-month follow-up (60.0\% vs $69.4 \%, \mathrm{p}=0.03) .^{52}$

Two studies found significant increases in diabetes self-efficacy and significant decreases in diabetes distress and medication nonadherence. ${ }^{50} 51$ Another study found no change in diabetes self-efficacy, diabetes distress or medication non-adherence. ${ }^{52} \mathrm{~A}$ significant decrease in depressive symptoms and binge drinking, but no effect on smoking or illicit drug use, was observed in one study. ${ }^{51}$ Despite other positive changes observed, the same study found no change in number of hospitalisations or emergency department visits. Similarly, Kennedy et al found significant increases in self-esteem and emotional wellbeing, but no change in self-reported quality of life. ${ }^{48}$

\section{Barriers and facilitators to intervention implementation}

The most commonly mentioned facilitators of implementation described characteristics of interventions that kept costs low. The majority of the included studies described reliance on food pantry/bank staff members or volunteers for various aspects of intervention implementation, ${ }^{47} 4850-52$ and one study described the relatively low cost of the foods that were provided to the participants. ${ }^{51}$ Studies described aspects of the intervention that increased convenience for participants (ie, locating a mobile pantry in the target community ${ }^{48}$ or scheduling education sessions during food distributions ${ }^{52}$ ) or for the implementation site (ie, allowing food banks to tailor the intervention to fit their capacities and workflow ${ }^{50}$ or allowing the pantry to identify potential participants). ${ }^{51}$ Two studies noted that they provided incentives to participants for completing components of the intervention or data collection events, which likely improved retention. ${ }^{49}{ }^{51}$ Likewise, two studies described leveraging participating organisations to facilitate implementation (eg, involving a multilevel, multiorganisation cancer coalition in implementation ${ }^{47}$ or allowing participating food banks flexibility to select specific food pantries as implementation sites). ${ }^{50}$ Other facilitators included reliance on an evidence-based intervention rather than developing a new intervention, ${ }^{47}$ minimising the level of cooking skills required for participants to complete intervention activities, ${ }^{49}$ and excluding potential participants who would have required home-delivered meals or special diets in order to participate. ${ }^{51}$

Compared with the descriptions of implementation facilitators, the studies' descriptions of implementation barriers were more heterogeneous. A commonly mentioned barrier to implementation intervention was the difficulty of recruiting and retaining food pantry clients as participants. One study noted that some eligible participants were not recruited because of the difficulty in having volunteers available at all hours the pantries were open. ${ }^{47}$ Another study described the difficulty of retaining participants drawn from a particularly mobile population. ${ }^{50} \mathrm{~A}$ third study indicated that only a minority of participants completed all components of a multicomponent intervention. ${ }^{52}$

Barriers related to organisational capacities were noted across studies. One study noted the difficulties inherent in implementing an intervention across a heterogeneous group of food pantries and food banks, ${ }^{50}$ and another study described how an intervention partner had only recently begun working with clients with type 2 diabetes. ${ }^{51} \mathrm{~A}$ third study described how distribution of the intervention food 
packages was limited by the capacity of participating food banks, resulting in large twice-monthly packages that participants found difficult to transport. ${ }^{52}$ The same study noted that participants still had access to non-intervention food from the food pantries, potentially diluting the effects of the intervention food. ${ }^{52}$ Another study noted that-given the funding, access to special pricing and staffing required to implement the intervention as part of the study-implementation of a sustainable version of the intervention would have been difficult. ${ }^{48}$ For one study, no implementation barriers were mentioned. ${ }^{49}$

\section{DISCUSSION}

This scoping review identified six studies that implemented a disease prevention or management intervention with at least one biometric outcome in a food pantry or food bank and focused on food pantry or food bank clients. This finding indicates a lack of research focused on evaluating disease prevention or management interventions using biometric indicators in this non-traditional research setting. However, these six studies employed a diverse range of intervention approaches to manage/ prevent a relatively wide range of chronic diseases. Likewise, the studies employed a range of biometric indicators, including BMI, body weight, HbAlc, glycaemic control, waist circumference, blood pressure, fasting glucose and diagnosed cancers. Although results were not uniform across studies, studies demonstrated that food pantry and food bank-based interventions can be associated with changes in biometric outcomes such as body weight, BMI and HbAlc.

Among the reviewed studies, BMI appears to have been the biometric outcome most sensitive to change in the typical intervention timeframe of 6 months. Of the three studies that reported baseline and follow-up BMI measures, all three noted significant reductions; however, Palar et al found significant reductions only for the subset of participants with type 2 diabetes, not for those with HIV. This is likely to be a result of the three interventions specifically targeting overweight/obese individuals and/ or those with type 2 diabetes.

Studies reporting significant changes in biometric outcomes provided education (eg, nutrition, cooking, diabetes self-management) in addition to healthier foods, suggesting providing food pantry clients with healthier food alone may not be sufficient to produce clinically or statistically significant changes in biometric outcomes. Finally, studies that documented good retention/high engagement, appeared more successful in effecting change compared with those that suffered high loss to follow-up/low rates of engagement.

These six studies also highlighted the racial and ethnic diversity of the population of food pantry and food bank clients who participated in these interventions, while also demonstrating that these populations of participants may be more likely to include women than men. These findings are unsurprising, given studies examining food insecurity and food pantry utilisation at the national level have consistently documented higher rates of food insecurity and food pantry utilisation among women than men and among blacks, Hispanics and 'other' races/ethnicities than whites. ${ }^{124}$ Given the small number of eligible studies, it is difficult to draw conclusions with respect to the presence or absence of heterogeneity of intervention effectiveness by sex or race/ethnicity. Collectively, the studies documented several barriers and facilitators that the research teams encountered as they implemented the interventions, particularly with respect to the costliness and sustainability of interventions, the convenience for participants or partner sites, and recruitment and retention of participants.

\section{Future research}

Health researchers seeking to conduct interventions in food pantries or food banks should address the barriers and facilitators highlighted in this review. For example, researchers should be aware of the staffing and training needs that will be required for successful implementation. The majority of food pantries rely on volunteers to conduct normal operations, so researchers must consider the extent to which the interventions' demands on staff time are reasonable or sustainable. Compensating food pantries and food banks for staff time devoted to intervention activities should be considered.

In addition, as promising disease prevention and management interventions are identified, more rigorous evaluations are warranted. Only two studies in the present review included control arms or random assignment. Likewise, the reviewed studies highlight the need to emphasise tracking retention of participants and participants' engagement with the interventions. Findings from the reviewed studies may point towards investigating the efficacy of alternative methods of distribution of nutrition education and healthier foods, such as home meal delivery. In addition, given the range of complexity of the reviewed interventions, researchers conducting similar studies should consider assessing the cost-effectiveness of their interventions. Also, given that many of these interventions are likely to affect multiple household members (ie, not only the individual research participant), researchers should consider examining any spillover effects that may be experienced by other household members as a result of the interventions. Finally, given the racially/ethnically diverse client base served by food pantries and food banks, future research may be warranted to identify the efficacy of providing culturally tailored disease prevention or management support.

\section{Limitations}

This scoping review has important limitations. First, the exclusion of studies that lacked biometric outcomes (eg, studies for which the outcomes comprised improvements in dietary quality) resulted in the exclusion of some studies that implemented interventions in food pantries and food banks and that therefore could have 
provided relevant information about implementation barriers and facilitators. Second, this review focused on published peer-reviewed studies and did not include grey literature (eg, conference abstracts, unpublished manuscripts, organisational reports). For this reason, otherwise eligible studies or outcomes may have been excluded from the review due to researchers' or journals' tendencies to publish positive results, whether at the study level (ie, failing to write or publish manuscripts that do not demonstrate statistically significant effects) or the outcome level (ie, omitting or minimising description of indicators for which no significant effects were demonstrated). Third, there was a notable lack of detail in several of the studies regarding specific characteristics of the food pantries or food banks at which the interventions were implemented and the authors did not contact the authors of the included studies to obtain further information regarding intervention implementation. Fuller descriptions of the implementing food pantries and food banks would have enhanced the usefulness of this review's description of implementation barriers and facilitators, which are likely to be of interest to researchers who plan studies in food pantries and food banks. Fourth, the auto alerts the authors received from November 2017 to August 2018 were implemented only for the MEDLINE database and not for other databases. It is possible the authors may have identified additional articles for inclusion in the review if the auto alerts had been expanded to the original search's entire set of databases.

\section{CONCLUSIONS}

To our knowledge, this is the first review to examine the peer-reviewed literature related to disease prevention and disease management interventions conducted in food pantries and food banks. The six studies identified in this review include a range of biometric indicators targeted by disease prevention and management interventions implemented in food pantries and food banks. These studies document a range of important barriers and facilitators to successful implementation of these interventions. However, the small number of eligible studies limits the evidence available to evaluate whether these interventions' effects vary as a function of participant demographic characteristics. More broadly, the small number of eligible studies suggests that more studies must be done before there can be a useful systematic review of the effectiveness of disease prevention or management interventions with at least one biometric outcome in a food pantry or food bank and focused on food pantry or food bank clients. Given the high number of households who obtain food from food pantries, ${ }^{2}$ as well as the chronic health conditions associated with food insecurity and inadequately nutritious dietary patterns, ${ }^{9}{ }^{12}$ the results of this scoping review underscore the need for additional high-quality research focused on disease management and prevention in this setting and with this population.
Contributors CRL conceived and designed the review, performed data acquisition, extraction, and synthesis, and drafted and revised the manuscript. BR contributed to the conception and design of the review, led data acquisition, extraction and synthesis, and drafted and revised the manuscript. SCS built search strategies with guidance from CRL and BR conducted database searches, drafted the Methods section and critically revised the manuscript. PAM contributed to the conception and design of the review and critically revised the manuscript. All co-authors reviewed and approved the submitted version.

Funding Support for this study was provided by a Translational Research Institute grant (\#1U54TR001629-01A1) from the National Center for Translational Sciences of the National Institutes of Health (NIH). The writing of this article was partially supported by the National Institute of General Medical Sciences of the NIH (\#P20GM109096). The content of this paper is solely the responsibility of the authors and does not necessarily represent the official views of the funders.

Competing interests None declared.

Patient consent for publication Not required.

Provenance and peer review Not commissioned; externally peer reviewed.

Data availability statement Final search strategies from all databases may be requested from the corresponding author (CRL).

Open access This is an open access article distributed in accordance with the Creative Commons Attribution Non Commercial (CC BY-NC 4.0) license, which permits others to distribute, remix, adapt, build upon this work non-commercially, and license their derivative works on different terms, provided the original work is properly cited, appropriate credit is given, any changes made indicated, and the use is non-commercial. See: http://creativecommons.org/licenses/by-nc/4.0/.

\section{REFERENCES}

1. Coleman-Jensen A, Rabbitt M, Gregory C, et al. Household Food Security in the United States in 2017. Washington, DC: U.S. Department of Agriculture, Economic Research Service, 2018.

2. Coleman-Jensen A, Rabbitt M, Gregory C, et al. Statistical Supplement to Household Food Security in the United States in 2017. Washington, DC: U.S. Department of Agriculture, Economic Research Service, 2018.

3. Ohls J, Saleem-Ismail F, Cohen R. The Emergency Food Assistance System - Findings From the Provider Survey, Volume II: Final Report Mathematica Policy Research, Inc. for the Food and Rural Economics Division, Economic Research Service, U.S. Department of Agriculture; 2002.

4. Weinfield N, Mills G, Borger C, et al. Hunger in America 2014: national report prepared for feeding America. Chicago, IL: Feeding America, 2014

5. Gundersen C, Ziliak JP. Food insecurity and health outcomes. Health Aff 2015;34:1830-9.

6. Tarasuk V, Cheng J, de Oliveira C, et al. Association between household food insecurity and annual health care costs. Can Med Assoc J 2015;187:E429-E436.

7. Berkowitz SA, Seligman HK, Meigs JB, et al. Food insecurity, healthcare utilization, and high cost: a longitudinal cohort study. Am J Manag Care 2018;24:399-404.

8. World Health Organization. Diet, Nutrition and the Prevention of Chronic Diseases: Report of a Joint WHO/FAO Expert Consultation. Geneva: World Health Organization, 2003.

9. Dietary Guidelines Advisory Committee. Scientific report of the 2015 dietary guidelines Advisory Committee: Advisory report to the Secretary of health and human services and the Secretary of agriculture. Washington, DC: United States Department of Agriculture, Agricultural Research Service, 2015.

10. Seligman HK, Bindman AB, Vittinghoff $E$, et al. Food insecurity is associated with diabetes mellitus: results from the National health examination and nutrition examination survey (NHANES) 1999-2002. $J$ Gen Intern Med 2007;22:1018-23.

11. Laraia BA. Food insecurity and chronic disease. Adv Nutr 2013:4:203-12.

12. Gregory CA, Coleman-Jensen A, Insecurity F. Chronic Disease, and Health Among Working-Age Adults. Washington, DC: United States Department of Agriculture, Economic Research Service, 2017.

13. Helmick M, Smith TM, Parks CA, et al. Food insecurity increases odds of diabetes and hypertension, not obesity in medically underserved region. J Hunger Environ Nutr 2018:e1-12.

14. Pan L, Sherry B, Njai R, et al. Food insecurity is associated with obesity among US adults in 12 states. $J$ Acad Nutr Diet 2012;112:1403-9. 
15. Franklin B, Jones A, Love D, et al. Exploring mediators of food insecurity and obesity: a review of recent literature. $J$ Community Health 2012;37:253-64.

16. Rasmusson G, Lydecker JA, Coffino JA, et al. Household food insecurity is associated with binge-eating disorder and obesity. Int $J$ Eat Disord 2019;52:28-35.

17. Seligman HK, Laraia BA, Kushel MB. Food insecurity is associated with chronic disease among low-income NHANES participants. $J$ Nutr 2010;140:304-10.

18. Shin J-I, Bautista LE, Walsh MC, et al. Food insecurity and dyslipidemia in a representative population-based sample in the US. Prev Med 2015;77:186-90.

19. Okechukwu CA, El Ayadi AM, Tamers SL, et al. Household food insufficiency, financial strain, Work-Family spillover, and depressive symptoms in the working class: the work, family, and health network study. Am J Public Health 2012;102:126-33.

20. Wang EA, McGinnis KA, Goulet J, et al. Food insecurity and health: data from the Veterans aging cohort study. Public Health Rep 2015;130:261-8.

21. Berkowitz SA, Baggett TP, Wexler DJ, et al. Food insecurity and metabolic control among U.S. adults with diabetes. Diabetes Care 2013;36:3093-9.

22. Grilo SA, Shallcross AJ, Ogedegbe G, et al. Food insecurity and effectiveness of behavioral interventions to reduce blood pressure, New York City, 2012-2013. Prev Chronic Dis 2015;12:E16.

23. Aibibula W, Cox J, Hamelin A-M, et al. Association between food insecurity and HIV viral suppression: a systematic review and metaanalysis. AIDS Behav 2017;21:754-65.

24. Tarasuk V, Mitchell A, McLaren L, et al. Chronic physical and mental health conditions among adults may increase vulnerability to household food insecurity. J Nutr 2013;143:1785-93.

25. Afulani PA, Coleman-Jensen A, Herman D. Food insecurity, mental health, and use of mental health services among nonelderly adults in the United States. J Hunger Environ Nutr 2018:1-22.

26. Martin KS, Wu R, Wolff M, et al. A novel food pantry program: food security, self-sufficiency, and diet-quality outcomes. Am J Prev Med 2013:45:569-75.

27. Wilson NL, Just DR, Swigert J, et al. Food pantry selection solutions: a randomized controlled trial in client-choice food pantries to nudge clients to targeted foods. J Public Health 2017;39:366-72.

28. Ippolito MM, Lyles CR, Prendergast K, et al. Food insecurity and diabetes self-management among food pantry clients. Public Health Nutr 2017;20:183-9.

29. Kaiser BL, Thomas GR, Bowers BJ. A Case Study of Engaging Hardto-Reach Participants in the Research Process: Community Advisors on Research Design and Strategies (CARDS) ${ }^{\circ}$. Res Nurs Health 2017;40:70-9.

30. Simmet A, Depa J, Tinnemann P, et al. The Dietary Quality of Food Pantry Users: A Systematic Review of Existing Literature. J Acad Nutr Diet 2017:117:563-76.

31. Simmet A, Depa J, Tinnemann P, et al. The Nutritional Quality of Food Provided from Food Pantries: A Systematic Review of Existing Literature. J Acad Nutr Diet 2017:117:577-88.

32. Strimbu K, Tavel JA. What are biomarkers? Curr Opin HIV AIDS 2010;5:463-6.

33. Williamson PR, Altman DG, Bagley $\mathrm{H}$, et al. The comet Handbook: version 1.0. Trials $2017 ; 18: 280$.
34. Moher D, Liberati A, Tetzlaff J, et al. Preferred reporting items for systematic reviews and meta-analyses: the PRISMA statement. PLoS Med 2009;6:e1000097.

35. Moher D, Shamseer L, Clarke M, et al. Preferred reporting items for systematic review and meta-analysis protocols (PRISMA-P) 2015 statement. Syst Rev 2015;4:1.

36. Shamseer L, Moher D, Clarke M, et al. Preferred reporting items for systematic review and meta-analysis protocols (PRISMA-P) 2015: elaboration and explanation. BMJ 2015;349:g7647.

37. Tricco AC, Lillie E, Zarin W, et al. PRISMA extension for scoping reviews (PRISMA-ScR): checklist and explanation. Ann Intern Med 2018;169:467-73.

38. Arksey H, O'Malley L. Scoping studies: towards a methodological framework. Int J Soc Res Methodol 2005;8:19-32.

39. Levac D, Colquhoun H, O'Brien KK. Scoping studies: advancing the methodology. Implementation Sci 2010;5

40. Long CR, Rowland B, Steelman SC, et al. Outcomes of disease prevention and management interventions in food pantries and food banks: protocol for a scoping review. BMJ Open 2017;7:e018022.

41. Counsell $\mathrm{C}$. Formulating questions and locating primary studies for inclusion in systematic reviews. Ann Intern Med 1997;127:380-7.

42. Robinson KA, Saldanha IJ, McKoy NA. Frameworks for Determining Research Gaps During Systematic Reviews. Rockville, MD: Agency for Healthcare Research and Quality, 2011.

43. Matchar DB. Introduction to the Methods Guide for Medical Test Reviews. In: Chang SM, Matchar DB, Smetana GW, et al, eds. Methods guide for medical test reviews. Rockville, MD: Agency for Healthcare Research and Quality, 2012.

44. Lorenzetti DL, Ghali WA. Reference management software for systematic reviews and meta-analyses: an exploration of usage and usability. BMC Med Res Methodol 2013;13:141.

45. Ouzzani M, Hammady H, Fedorowicz Z, et al. Rayyan-a web and mobile APP for systematic reviews. Syst Rev 2016;5:210.

46. Damschroder LJ, Lowery JC. Evaluation of a large-scale weight management program using the consolidated framework for implementation research (CFIR). Implementation Sci 2013;8.

47. Bencivenga $\mathrm{M}$, DeRubis $\mathrm{S}$, Leach $\mathrm{P}$, et al. Community partnerships food pantries, and an evidence-based intervention to increase mammography among rural women. J Rural Health 2008;24:91-5.

48. Kennedy BM, Champagne CM, Ryan DH, et al. The "Rolling Store:" an economical and environmental approach to the prevention of weight gain in African American women. Ethn Dis 2009;19:7-12.

49. Flynn MM, Reinert S, Schiff AR. A six-week cooking program of plant-based recipes improves food security, body weight, and food purchases for food pantry clients. J Hunger Environ Nutr 2013;8:73-84.

50. Seligman HK, Lyles C, Marshall MB, et al. A pilot food bank intervention featuring diabetes-appropriate food improved glycemic control among clients in three states. Health Aff 2015;34:1956-63.

51. Palar K, Napoles T, Hufstedler LL, et al. Comprehensive and medically appropriate food support is associated with improved HIV and diabetes health. J Urban Health 2017;94:87-99.

52. Seligman HK, Smith M, Rosenmoss S, et al. Comprehensive diabetes self-management support from food banks: a randomized controlled trial. Am J Public Health 2018;108:1227-34. 